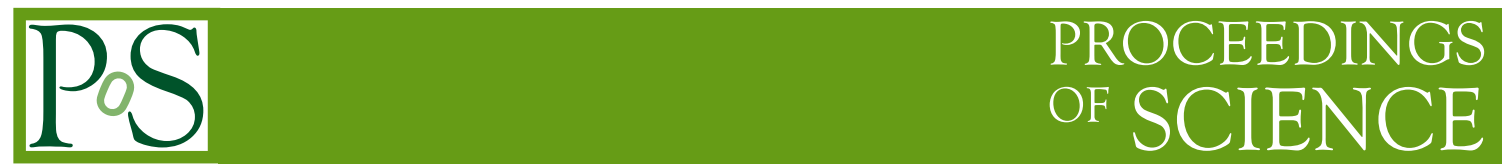

\title{
Search for long-lived particles in LHCb
}

Marcin Kucharczyk on behalf of LHCb collaboration ${ }^{a, *}$

${ }^{a}$ Henryk Niewodniczanski Institute of Nuclear Physics PAN,

Krakow, Poland

E-mail: marcin.kucharczyk@cern.ch

The present document focuses on the recent searches for the existence of heavy particles beyond the Standard Model at LHCb, exploiting its unique forward acceptance, high-precision tracking and excellent particle identification. Searches for long-lived particles decaying into jet pairs or semileptonically, together with the lepton-flavour-violating decays of Higgs-like boson, are discussed.

The Eighth Annual Conference on Large Hadron Collider Physics-LHCP2020

25-30 May, 2020

Paris, France

${ }^{*}$ Speaker 


\section{Introduction}

Many models of new physics predict the existence of new, long-lived particles (LLP), which would appear with a very distinctive experimental signature. Physics beyond the Standard Model (SM) via the production of such particles has been searched for at the LHC without success so far. The results provided by the $\mathrm{LHCb}$ experiment contribute to these searches. The LHCb detector [1] is a single-arm spectrometer fully instrumented in the forward direction, $2<\eta<5$, equipped with a high-precision tracking and particle identification systems. It provides efficient track and vertex reconstruction and an excellent particle identification at small angles with respect to the beam direction, complementary to the general purpose LHC detectors. It proved to be a unique tool to study a broad spectrum of physics phenomena in the forward region, providing high precision results at the highest available collision energies. It is naturally suited for the search of beyond Standard Model long-lived particles in a broad range of mass and lifetime.

The latest $\mathrm{LHCb}$ results on searches for long-lived particles decaying into jet pairs in the context of the Hidden Valley (HV) model [2], or semileptonically within the minimal supergravity (mSUGRA) with $R$-Parity and baryon number violating couplings [3], together with the leptonflavour-violating decays of Higgs-like boson, are reviewed.

\section{Search for long-lived particles decaying into jet pairs}

The Hidden Valley model predicts the existence of a new non-abelian gauge group consisting of so-called $v$-particles, hidden at large energy scales. The hidden region may be accessible at the LHC, through the decay of hidden particles into SM particles through heavy mediators. In particular, it predicts the SM-like Higgs boson to decay into the combinations of massive longlived particles $\left(\pi_{v}\right.$ 's) that decay mostly to a $b \bar{b}$ pair. In the case of Hidden Valley model the event topology is a set of displaced vertices, distant from the primary vertex and the beam axis. A search for $v$-particles has been performed using a dataset of proton-proton collisions at 7 and $8 \mathrm{TeV}$, corresponding to an integrated luminosity of $2 \mathrm{fb}^{-1}$ [4]. The approximate sensitivity range of this analysis covers long-lived particle lifetimes from 2 to $500 \mathrm{ps}$ and masses $\left(m_{\pi_{v}}\right)$ between 25 and $50 \mathrm{GeV} / \mathrm{c}^{2}$. As in most of the cases only one Hidden Valley pion decays within the $\mathrm{LHCb}$ acceptance, the signature of the signal is one displaced vertex with two associated heavy flavour jets. The selection is based on the decays that produce a sufficient number of tracks in the vertex detector, with a cut on the radial distance of the displaced vertex to the beam axis. In order to reduce the background related to the secondary interactions with the detector material, vertices inside a certain veto region around the LHCb vertex detector are excluded. The signal yield is determined from the fit to the di-jet invariant mass distribution, and it is modelled by the Gaussian distribution with the parameters taken from the simulated signal. Displaced vertices from heavyflavour decays and secondary interactions with detector material are the main source of background. It is modelled by the convolution of exponential distribution with a bifurcated Gaussian. After the selection, no candidates are found and 95\% CL upper limits are set for the production cross section. Figure 1 shows the cross-section upper limits as a function of lifetime for hidden-valley models with different $\pi_{v}$ masses and decay modes. 


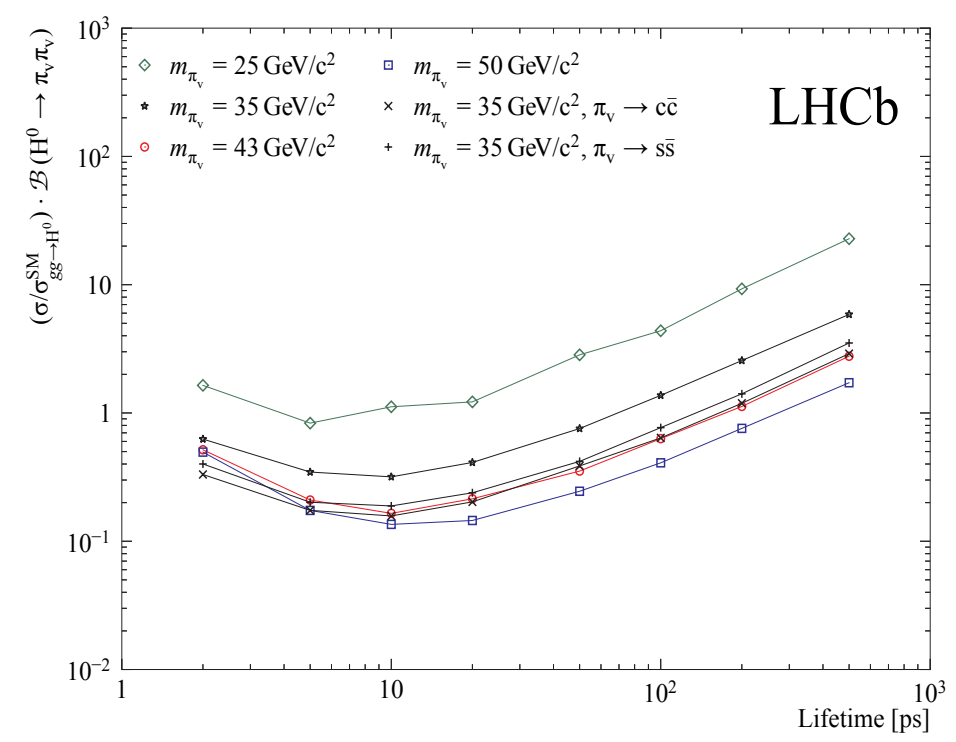

Figure 1: Upper limits at 95\% CL versus lifetime for different $\pi_{v}$ masses and decay modes. The decay $\pi_{v} \rightarrow b \bar{b}$ is assumed, unless specified otherwise. Figure taken from [4].

\section{Search for long-lived particles decaying semileptonically}

In the framework of weak scale supersymmetry with $R$-parity violation the lightest super partner may decay into SM particles. In particular, it has been proposed that the lightest neutralino $\widetilde{\chi}_{1}^{0}$ may decay into a muon and two jets, and the SM-like Higgs boson decays into two neutralinos. In this context the event topology is a displaced vertex with several tracks including high $p_{T}$ muon. A search for long-lived particles decaying semileptonically has been performed using a dataset of proton-proton collisions at 7 and $8 \mathrm{TeV}$, corresponding to an integrated luminosity of $3 \mathrm{fb}^{-1}$ [5]. The approximate sensitivity range of this analysis covers long-lived particle lifetimes from 5 to $100 \mathrm{ps}$ and masses between 20 and $200 \mathrm{GeV} / \mathrm{c}^{2}$. Signal events are looked for by requiring a displaced high multiplicity vertex with one associated isolated $p_{T}$ muon. The final selection is based on multi-variate analysis, where the radial distance of the displaced vertex to the beam axis is the most discriminant variable. In order to reduce the background related to the secondary interactions with the detector material, the material veto is applied. The signal yield is determined with an extended maximum likelihood fit to the distribution of the reconstructed long-lived particle mass, with the shape of the signal component taken from the simulated models. No simulated background survives after applying multi-variate based selection, so the data-driven method is used to determine the background shape. As no excess above the background expectation is observed, the upper limits at $95 \% \mathrm{CL}$ on the production cross-sections times branching fractions are determined, for different theoretical models and for several mass and lifetime hypotheses.

\section{Lepton-flavour-violating decays of Higgs-like boson}

Decays with the charged-lepton flavour violation are forbidden in the Standard Model. Thus, such an observation would be a clear signal for the physics beyond the Standard Model. Such 
processes are predicted by several theoretical models, in particular supersymmetric models [7] or composite Higgs models [8]. The analysis concerns the Higgs boson decaying into muon and $\tau$ leptons, so the signature of the signal is a prompt muon and displaced $\tau$ lepton. It has been performed using a dataset of proton-proton collisions at $8 \mathrm{TeV}$, corresponding to an integrated luminosity of $2 \mathrm{fb}^{-1}$ [6]. The analysis is separated into four channels depending on the final state of the $\tau$ lepton decay, and it is performed for Higgs boson masses from 45 to $195 \mathrm{GeV} / \mathrm{c}^{2}$. The main sources of background are the $Z \rightarrow \tau \tau$ decays, heavy flavour production from QCD processes and electroweak boson production accompanied by jets. The signal yield is determined from extended likelihood fit to the binned invariant mass distribution for the $\mu \tau$ candidates. The fit results for all studied Higgs masses are compatible with no signal, thus the upper limits at 95\% CL on the cross-section times branching fraction are set. In a particular case, assuming the SM Higgs boson production cross-section, the observed exclusion limit is below $26 \%$.

\section{Conclusions}

LHCb has used the large data sample available from Run I to perform studies of processes beyond the Standard Model in a complementary phase space with respect to the general purpose LHC detectors. The searches for long-lived particles decaying into jet pairs or semileptonically have been presented, together with the lepton-flavour-violating decays of Higgs-like boson that LHCb can also investigate. All the results presented are consistent with the Standard Model expectations and demonstrate the excellent capabilities of the the LHCb detector as a high-resolution forward spectrometer. The upcoming upgrades of the LHCb detector with improved triggering, tracking and vertexing can produce more competitive upper limits for long-lived particles with low masses and lifetimes. A better impact parameter resolution due to the upgraded vertex locator, a better knowledge of material interactions, and improved jet reconstruction algorithms, will play a crucial role in order to achieve unprecedent sensitivities for this kind of searches.

\section{References}

[1] LHCb collaboration, A. A. Alves Jr. et al., JINST 3 (2008) S08005.

[2] M. J. Strasser, K. M. Zurek, Phys. Lett. B651 (2007) 374.

[3] L. M. Carpenter, D. E. Kaplan, E. J. Rhee, Phys. Rev. Lett. 99 (2007) 211801.

[4] LHCb collaboration, R. Aaij et al., Eur. Phys. J. C77 (2017) 812.

[5] LHCb collaboration, R. Aaij et al., Eur. Phys. J. C77 (2017) 224.

[6] LHCb collaboration, R. Aaij et al., Eur. Phys. J. C78 (2018) 1008.

[7] J. L. Diaz-Cruz, J. J. Toscano, Phys. Rev. D62, (2000) 116005.

[8] K. Agashe, R. Contino, Phys. Rev. D80, (2009) 075016. 